Experiences of psychiatrists assessing under 18s on an "all age" rota. An evaluation of the current service

Nicoleta Clarisa Turtoi ${ }^{1 \star}$, Martin Ansell ${ }^{1}$, Trinisha Govender ${ }^{1}$ and Helen Bould ${ }^{2}$

${ }^{1}$ Gloucestershire Health and Care NHS Foundation Trust and

${ }^{2}$ Gloucestershire Health and Care NHS Foundation Trust, Centre for Academic Mental Health, University of Bristol

${ }^{*}$ Corresponding author.

doi: 10.1192/bjo.2021.830

Aims. Gloucestershire Health Care Trust operates an "all age" out of hours on-call rota, staffed by Registrars and Consultants who cross-cover all psychiatric sub-specialities. Our aim was to understand the challenges faced by psychiatrists of different professional backgrounds when assessing under 18 year olds in a health-based Place of Safety (POS).

Method. We circulated a survey to all psychiatrists on the on-call rota, to gather quantitative and qualitative information on the challenges posed by assessments of under-18s in a POS and assess whether an under-18 specific policy was needed.

Result. Out of the 50 psychiatrists invited, 27 completed this survey (during February 2021). 33.3\% of respondents reported that they had completed a POS assessment of an under 18-year-old when a Consultant Child and Adolescent Psychiatrist/Registrar was not available to join. $33.3 \%$ of respondents had been asked to complete such an assessment as the sole psychiatrist joining the AMHP, with $24 \%$ of those respondents reporting feeling uncomfortable doing these assessments without a second doctor present, and an additional $24 \%$ feeling comfortable only sometimes. $48.1 \%$ of the doctors surveyed did not invite parents or carers to take part in the assessment, despite this being considered best practice, citing reasons such as: being unaware that this was a possibility, assessments conducted at unsociable hours, safeguarding concerns involving the parents, and social distancing in the context of COVID-19. 41\% of respondents had assessed vulnerable young people (children looked after or with a diagnosis of an autism spectrum condition) in the POS and reported that these assessments posed significant challenges to safe discharge planning and identifying appropriate placements. $81.5 \%$ felt that a tailored policy for young people was needed. Qualitative findings suggest psychiatrists think such a policy should include clear procedures (flowcharts), potential outcome scenarios/ options for safe discharge, referral criteria for CAMHS, contact details for key staff members, a handover protocol. Respondents felt a shared policy with all stakeholders (AMHP service, Mental Health Services, police) was required.

Conclusion. Our survey highlights the challenges for non-CAMHS specialists assessing under $18 \mathrm{~s}$ in a health-based POS and the need for an under-18 policy to support safe practice. Major themes will be further explored in a focus group to guide policy development.

\section{Assessing DNA rates within first time psychiatric referrals and the extent to which DNA rates are reduced by an SMS reminder service}

Henry Coates

University of Birmingham

doi: 10.1192/bjo.2021.831

Aims. 1) To assess the average wait time for patients to be offered an appointment and to establish any correlations between longer waiting times and 'Did not attend (DNA)' rates 2) To assess the number of patients who have opted into the text message appointment reminder service and whether this had an effect on DNA rates.

Background. Research has indicated that the Did Not Attend (DNA) rate in Psychiatry is estimated at 20\%, twice that of other medical specialties (1). With NHS Digital estimating that DNAs cost the NHS $£ 1$ Billion per annum, there has been much interest in reducing the rate of DNAs within Psychiatry (2). Findings have shown that short waiting times are associated with higher rates of attendance (3). In addition, poor appointment attendance within Psychiatry is also associated with increased disease severity and higher rates of hospital admission (4).

Method. We conducted retrospective data collection on 99 patients referred to Professor Oyebode between January 2018 and August 2019. Our data collection involved assessing time the referral was received, time to first appointment and the patient's communication preference (e.g. whether they opted in to the SMS alert service). All data collection was conducted through use of RIO and coded/ammonized into a Excel spreadsheet. No sampling methods were employed and our population only consisted of first-time referrals to Professor Oyebodes clinic.

Result. 1) We found no correlation between a longer waiting time to first appointment and an increased DNA rate.

2) All patient waiting times between 1st January - 31st August were within the maximum limit set by national guidelines

3) Opting into the text messaging service remains severely low. Of the patients audited, 95\% had not completed a communication preference form. Overall, it is still unclear whether the text messaging service has a positive impact on DNA rates.

Conclusion. Our data have shown no significant correlation between a longer waiting time and an increased DNA rate for first time Psychiatry appointments. Secondly, we have concluded that between the audited period, waiting times were still within the maximum 18 week wait set by the Mental Health Standards. Finally, we can conclude that uptake of the text messaging service remains very low at $4 \%$. Due to a limited sample size of only 4 patients, it is still unclear from this audit whether opting into the text messaging services will have a positive decrease on the number of DNA's.

'Foreseeing well-being': developing a physical health strategic vision across a large mental health trust 'foreseeing well-being': developing a physical health strategic vision across a large mental health trust

Bethany Cole*, Nwaorima Kamalu and Kyra Neubauer Avon and Wiltshire Mental Health Partnership NHS Trust ${ }^{\star}$ Corresponding author.

\section{doi: 10.1192/bjo.2021.832}

Aims. Statistically, suicide is less than half as deadly as poor physical health for people with severe mental illnesses (SMI). For every 1000 SMI patients, diseases such as diabetes cause 10-20,000 'years of life lost' compared to 4,000 'years of life lost' to suicide. National charity Rethink dubbed the failure of the NHS to act on this as tantamount to "lethal discrimination".

We aim to reform the physical health care provision for service users under the care of Avon and Wiltshire Mental Health Partnership NHS Trust (AWP).

Method. To evaluate the current service within AWP, we combined data from a comprehensive audit of 106 inpatients, local Quality Improvement (QI) Projects, and qualitative feedback from a pilot Medical-Psychiatric Liaison Service (MPLS). 
Result. Key findings included:

High rates of physical comorbidities among psychiatric inpatients of all ages

Novel illnesses occurring during admissions

Evidence that patients are not receiving adequate physical healthcare from wider NHS

Junior doctors receiving inadequate support from Seniors and acute Hospital services when managing physical illnesses

Poor recording of cardiometabolic monitoring with few interventions delivered (even when indicated) and challenges finding relevant data in records.

During the MPLS pilot, a Consultant Physician provided virtual ward rounds and advisory sessions. 100\% of staff involved reported the service was beneficial for their clinical practice and patient outcomes.

Conclusion. Taking these findings and input from colleagues within AWP and nationally, we created a comprehensive strategic overview on how AWP can deliver high quality physical health care, detailing improvements to make across 5 key domains: Inpatient, Community, Workforce, Education and Information Technology (IT).

Presently, we are working with Clinical Commissioning Groups developing protocols clarifying roles and responsibilities across primary and secondary providers. We are standardising communication between AWP and primary care and expanding links with specialist secondary services (e.g. endocrinology and cardiology). We formed the BRIGHT (Better Recording of Information for Governance and Healthcare in the Trust) project workgroup alongside IT to build safer and more effective records systems.

Medium term recommendations include employing a full-time MPLS Consultant Physician, in addition to 'Physical Health and Wellbeing Workers' in all localities, Advanced Nurse Practitioners (working within structured physical care systems) and more allied health professionals (dieticians, speech therapists and physiotherapists).

In the long term, the new Physical Health, IT and QI working groups will maintain development of these proposals, improve training and supervision for clinicians, and achieve healthcare parity for patients across localities.

\section{The impact of COVID-19 on an inpatient mother and baby unit: a service evaluation}

Joanna Cranshaw*, Gertrude Seneviratne, Ranga Rao, Julia Ogunmuyiwa, Rebecca McMillin, Chukwuma Ntephe and Victoria Dain

South London and Maudsley NHS Foundation Trust

${ }^{\star}$ Corresponding author.

doi: $10.1192 /$ bjo.2021.833

Aims. Unique challenges have been faced by women in the perinatal period during the COVID-19 pandemic and the impact of this is compounded for women suffering from mental illness. This service evaluation looked at different aspects of the treatment pathway on a specialist inpatient psychiatric Mother and Baby Unit during the pandemic to identify what changes occurred.

Method. Data were collected for all admissions to the unit between January 2019 and October 2020, with the beginning of the pandemic being defined as on or after 1st March 2020. Information was collected retrospectively from electronic clinical notes on ethnicity, length of stay, diagnosis, mental health act use and restrictive practice, medication, psychology, occupational therapy and social services involvement.

Result. There were 114 admissions to the MBU during the study period. 4 were parenting assessments rather than acute psychiatric admissions and were excluded from the analysis, giving a sample of 110 women. 58\% (62/110) were classed as "pre-pandemic" and $43.6 \%$ (48/110) were "during pandemic". 95.45\% (105/110) of women were postpartum $4.55 \%$ (5/110) were pregnant. Mean length of stay was shorter during the pandemic at 44 days, compared to 61 pre-pandemic. There was greater use of the mental health act during the pandemic: only $43.75 \%$ of patients were informal throughout admission, compared to $70.97 \%$ prepandemic. Mean duration of detention was shorter at 25 days (32 pre-pandemic). Psychotic illness made up a greater proportion of diagnoses during the pandemic: $56 \%$ (27/48) compared to $44 \%(27 / 62)$ pre-pandemic. The next most common diagnostic group was mood and anxiety disorders, which made up 29\% (14/ 48 ) of diagnoses during the pandemic, but $43 \%$ (27/62) prepandemic. Outcomes as measured using the Health of the Nation Outcome Scale showed a mean improvement between admission and discharge of 6.65, compared to 5.15 pre-pandemic. HONOS scores were higher on admission during the pandemic (12.83, vs 10.88), suggesting a higher level of acuity.

Conclusion. During the COVID-19 pandemic on this Mother and Baby Unit, length of stay was shorter, a greater proportion of patients were detained under the mental health act (although length of detention was shorter) and psychotic illness was more prevalent. This study demonstrates that there were differences in this perinatal inpatient population during the pandemic and this may be a reflection on the wider impact of COVID-19 on perinatal mental health.

\section{Audit of shared-care lithium monitoring in a large rural GP practice}

Thomas Cranshaw

Cumbria, Northumberland Tyne and Wear Foundation Trust

doi: 10.1192/bjo.2021.834

Aims. To compare monitoring of lithium treatment with shared care lithium monitoring agreements in a large rural GP practice. Background. A 'near miss' event with a patient with drug induced long QT syndrome highlighted a need for an audit of lithium monitoring at a large rural GP practice.

The practice had entered into shared-care monitoring agreements with the local mental health care trust. Under these agreements, responsibility for physical monitoring of lithium treatment was assumed by the practice.

Method. Using audit functions built into the IT system, all patients at the practice who were currently prescribed lithiumcontaining medications were identified $(n=28)$. Individual monitoring standards were determined for each patient based on the shared care agreement. These varied depending on age and comorbidity. Monitoring data obtained from medical records was compared against the individualised monitoring requirement. Result. The key finding was that $26 \%$ of patients for whom annual ECGs were indicated according to the shared care agreement had received an ECG in the past year. $78 \%$ of patients had a lithium level recorded in the previous 3 months. $81 \%$ of patients had a renal function test within their monitoring requirements. 52\% of patients had lipid measurement in the previous year.

Conclusion. There is a great degree of heterogeneity in the extent to which shared care monitoring agreements are achieved. It is noted that those standards to which a Quality Outcome Framework incentive applied had a greater chance of being met. 